Ivánovics, G. \& NAGY, E. (1958). J. gen. Microbiol. 19, 407-418

\title{
Hereditary Aberrancy in Growth of Some Bacillus megaterium Strains
}

\author{
By G. IVANOVICS AND E. NAGY \\ Institute of Microbiology, Medical University, Szeged, Hungary
}

\begin{abstract}
SUMMARY : Two hundred strains of Bacillus megaterium recently isolated from soil were tested for an antagonistic effect on a phage-resistant strain of the same species. Fifty-one strains gave a zone of inhibition on a lawn of the indicator strain. This effect was increased by a small dose of ultraviolet radiation which also caused 41 other strains to show an antagonistic effect. Sixteen strains which were completely lysed after u.v. irradiation also produced confluent lysis of the indicator strain. When grown in liquid media, these strains gave irregular growth curves owing to partial lysis during the exponential phase. Lysis was almost complete when such cultures were incubated after exposure to small doses of u.v. radiation. Lysis was always associated with accumulation of the antibacterial principal previously named 'megacin'. Irregular growth in liquid media and production of megacin were strictly correlated. However, only 2 megacinogenic strains were lysogenic and liberated phage. Populations of megacinogenic strains were examined by replicaplating for the presence of non-megacinogenic mutants. Spore suspensions of 4 megacinogenic strains contained non-megacinogenic mutants in various proportions depending on the strain and the particular sample. Non-megacinogenic mutants could not be made to lyse by exposure to u.v. radiation; did not produce any antibacterial effects; and all showed normal patterns of growth in various liquid media. It is supposed that megacinogeny and aberrant growth, as well as inducibility by u.v. radiation, of some $B$. megaterium strains are governed by a single hereditary unit (gene). It cannot be decided at present whether or not this unit is a highly defective prophage.
\end{abstract}

Some strains of Bacillus megaterium are capable of producing an antibacterial substance of protein character which has been named 'megacin' (Ivánovics \& Alföldi, 1954, 1955). The production of this substance by growing bacteria is enhanced by irradiation with small doses of ultraviolet (u.v.) radiation which causes mass lysis and liberation of megacin. An interesting phenomenon appeared when organisms of a typical megacinogenic strain (no. 216) were grown with megacin-sensitive bacteria on the surface of solid media; plaques were formed which simulated the growth of lysogenic bacteria inoculated with an indicator strain. No phage particles could be detected in these plaques by either cultivation or electron microscopy (Ivánovics, Alföldi \& Lovas, 1957).

We found recently that a considerable proportion of Bacillus megaterium strains showed an unusual pattern of growth in liquid medium since, after an initial period of normal growth, the turbidity of the culture decreased owing to mass lysis of the organisms. The lytic phase was in many cases only transient, as the turbidity later increased. The growth curves of these aberrant strains were variable and inconsistent, for even the same inoculum might give different curves when grown in different media. Furthermore, the results obtained in individual experiments carried out under apparently identical 
conditions sometimes differed. The aim of the present investigation was to find out whether there is any relation between bacteriocinogeny and aberrant growth in $\boldsymbol{B}$. megaterium strains. A study was also made to estimate the frequency of aberrant strains in soil. It will be shown below that a hereditary character may be responsible for aberrant growth.

\section{METHODS}

The methods were in general those used by Ivánovics \& Alföldi (1955, 1957). Strains of Bacillus megaterium were isolated and identified as described by Ivánovics, Alföldi \& Széll (1957) and were maintained by subculture on yeast-extract peptone (YP) agar. Strain 216 was described by Ivánovics \& Alföldi (1955, 1957) and by Ivánovics, Alföldi \& Lovas (1957), and it was maintained on yeast-extract casein-digest (YDC) agar. A streptomycinresistant mutant $(216 \mathrm{~S}$ ) was obtained on streptomycin agar.

Phages. These were the same as those described by Ivánovics, Alföldi \& Széll (1957). 'Adapted (mutant) phages, highly specific for strains 216, 605 and 638 , respectively, were isolated in the usual way from either phage $\mathbf{M}_{1}$ or phage $\mathrm{M}$ ii. The phages selected for adaptation had a relatively low efficiency of plating (e.o.p.) on the above strains. The e.o.p. of phage $M_{1}$ was $10^{-6}$ on strain 216 and $2 \times 10^{-6}$ on strain 605 , when the values for adapted phages was taken as 1. Phage Mii had an e.o.p. of $10^{-5}$ on strain 638 before adaptation. The differences between the e.o.p. values of wild type and adapted phages provided a highly reliable means for identification of individual strains.

Nutrient media. By broth is meant horse meat broth containing $1 \%(\mathrm{w} / \mathrm{v})$ peptone (Richter, Budapest). Yeast extract peptone medium (YP) and yeast extract + enzymic digest of casein medium (YDC) have been described elsewhere (Ivánovics \& Alföldi, 1955, 1957). Solid media contained $1.5 \%$ (w/v) agar. Agar medium for the sporulation of Bacillus megaterium contained $0 \cdot 1 \%(w / v)$ enzymic digest of casein (Aminosol, Vitrum, Stockholm) as described by Tarr (1932).

Preparation of spore suspensions. Bacteria were grown either on Tarr's agar or on YP agar for several days at $37^{\circ}$. Spores formed in $95-98 \%$ of the organisms under these conditions. Spores were washed twice with saline and stored in the refrigerator.

Titration of megacin. A strain of Bacillus megaterium, Mut-C, which is resistant to megaterium phages, was used (Ivánovics, Alföldi \& Lovas, 1957). The indicator plates used for megacin titrations were also used in a screening test for iso-antagonistic (megacinogenic) strains. In this case, a drop of a young culture of the test strain was spotted on the surface of the indicator plate and irradiated with a high pressure u.v. lamp for 6 sec. before incubation.

The source of ultraviolet light. Two sources were used: a 'Hanau' highpressure lamp used at a distance of $25 \mathrm{~cm}$., as described by Ivánovics \& Alföldi (1955, 1957); and a low-pressure lamp, emitting $7 \mathrm{erg} . / \mathrm{sec} . / \mathrm{mm} .^{2}$ at $2537 \AA$. at $1 \mathrm{~m}$. on the optical axis.

Isolation of non-megacinogenic mutants from megacinogenic strains. Stock 


\section{Hereditary aberrancy in Bacillus megaterium}

spore suspensions or spores harvested from cultures grown from u.v.-irradiated spores were used. In the latter case, YP plates spread with $c .10^{7}$ spores were exposed to the high-pressure u.v. lamp for 160-240 sec. After incubating the plates at $37^{\circ}$ for 4-5 days, there were $c$. 30-200 colonies, consisting mainly of spores, which were washed off and stored in the refrigerator. This material is referred to below as 'u.v. spores'.

One-tenth $\mathrm{ml}$. samples of appropriate dilutions of spore suspensions, containing 40-70 colony-formers, were spread on several meat extract agar plates, the agar content being $2.5 \%$. After incubation overnight at $30^{\circ}$, the plates were placed in an incubator at $37^{\circ}$ and kept there until the colonies were 1.5-2 $\mathrm{mm}$. in diameter. These colonies were then tested by replica-plating (Lederberg \& Lederberg, 1952) to nutrient agar plates covered with an agar layer containing megacin-sensitive bacteria. Immediately after transfer of the test colonies, the indicator plates were exposed to the 'Hanau' lamp for 6 sec. After further incubation, megacinogenic colonies produced either clear plaques or colonies surrounded by a growth-free zone. Colonies which did not show an antibacterial effect were re-streaked from the test plate and retested several times using young cultures in YP medium. Only isolates which did not show any antagonistic effect were accepted as non-megacinogenic.

Identification of non-megacinogenic isolates. Bacillus megaterium strain 216 was identified by the serological specificities of its cell wall, using rabbit antiserum in an agglutination test (Ivánovics, 1955). Resistance to streptomycin was checked by streaking on agar containing $20 \mu \mathrm{g}$. streptomycin $/ \mathrm{ml}$. agar.

The phage sensitivity of mutants was tested by spotting decimal dilutions of the phages on $10^{7}$ organisms of the test strain incorporated in a soft agar layer. When highly specific 'adapted' phages were used, dilutions of the wildtype phage were included as a control.

\section{RESULTS}

The characteristics of aberrant Bacillus megaterium strains

Screening of strains by their iso-antagonistic effects. Iso-antagonistic effects between strains of Bacillus megaterium (Ivánovics \& Alföldi, 1955) served as markers for defining groups among strains recently isolated from soil. The criteria of an iso-antagonistic effect was that the strain should produce a zone of inhibition on the surface of an indicator plate seeded with a phage-resistant strain of B. megaterium. Two identical indicator plates, each spotted with a suspension of the test strain, were prepared. One was u.v. irradiated before incubation, while the second, which was not exposed to u.v. radiation, served as a control. The test strains were then classified by their colonial appearance, as follows: (1) no inhibition around the colony; (2) an inhibition zone not exceeding $2 \mathrm{~mm}$. in width; (3) a zone greater than $2 \mathrm{~mm}$. in width; and (4) a growth-free area of diameter $10-14 \mathrm{~mm}$. at the site of inoculation. Irradiation of the plates before incubation either induced or enhanced the iso-antagonistic effect with many of the strains. Table 1 gives 200 strains, recently isolated from 
soil, classified in this way. Sixteen strains gave maximum inhibition on the indicator plates and these form the main subject of the following study.

Table 1. Classification of 200 strains of Bacillus megaterium according to their iso-antagonistic effect

\begin{tabular}{|c|c|c|c|}
\hline \multicolumn{2}{|c|}{ No u.v. irradiation } & \multicolumn{2}{|l|}{ After u.v. irradiation } \\
\hline No effect & 149 & $\left\{\begin{array}{l}\text { No effect } \\
\text { Zone appeared } \\
\text { Clear plaque }\end{array}\right.$ & $\begin{array}{r}108 \\
38 \\
3\end{array}$ \\
\hline $\begin{array}{l}\text { Zone less than } \\
2 \mathrm{~mm} \text {. }\end{array}$ & 44 & $\left\{\begin{array}{l}\text { Same as without u.v. } \\
\text { Zone increased } \\
\text { Clear plaque }\end{array}\right.$ & $\begin{array}{r}5 \\
29 \\
10\end{array}$ \\
\hline $\begin{array}{l}\text { Zone broader than } \\
2 \mathrm{~mm} \text {. }\end{array}$ & 7 & $\left\{\begin{array}{l}\text { Same as without u.v. } \\
\text { Zone increased } \\
\text { Clear plaque }\end{array}\right.$ & $\begin{array}{l}\mathbf{0} \\
\mathbf{4} \\
\mathbf{3}\end{array}$ \\
\hline Total & 200 & & 200 \\
\hline
\end{tabular}

Cultivation of strains of Bacillus megaterium in liquid media. Media were inoculated from an overnight YP or YDC agar culture and growth of the organism followed by measuring the optical density of the cultures, aeration and turbidity measurements being carried out as described by Ivánovics \& Alföldi (1955, 1957). For technical reasons, the experiments were ended after $10 \mathrm{hr}$. A typical megacinogenic strain (no. 216; Ivánovics \& Alföldi, 1955,1957) was also studied. All the 17 strains which showed a marked iso-antagonistic effect in screening tests were grown several times in different liquid media during these investigations over a period of nearly two years. In one series of experiments, only 3 strains gave a normal curve, the remaining 14 being aberrant. By 'normal' growth, we mean logarithmic increase after a lag phase of $3 \mathrm{hr}$. The shapes of the aberrant curves were rather variable; curves from some typical experiments are shown in Figs. 1 and 2.

The growth curve of a given strain might vary markedly from one experiment to the next, although all experiments were carried out under the same conditions. Figure 3 shows variable results obtained with strain 605 . The variability of the growth curves should not be related to any detail of the experimental technique. However, the medium generally had a marked effect on the outcome of the experiment. Thus, some strains gave very aberrant curves when grown in YP medium while the same inoculum grew normally in another medium, such as YDC medium. We are not able to give a reasonable explanation for the observed discrepancies between individual experiments so that the lysis of these strains of Bacillus megaterium has at present to be attributed to an 'uncontrollable' factor or factors.

Eight strains which did not show an iso-antagonistic effect even after exposure to u.v. radiation were also included for comparison. All these strains gave normal growth in all media tested. Furthermore, aberrant growth could not be produced even by a small dose of u.v. radiation. Similar results were obtained with 6 strains which showed a moderate iso-antagonistic effect 


\section{Hereditary aberrancy in Bacillus megaterium}

(i.e. a zone of less than $2 \mathrm{~mm}$. diameter, not increased by u.v. irradiation). All the above observations support our assumption that a marked iso-antagonistic effect in the screening test was well correlated with aberrant growth in liquid medium. Also, the degree to which iso-antagonistic effects are enhanced by u.v. irradiation serves as an efficient marker for the isolation of strains exhibiting aberrant growth.

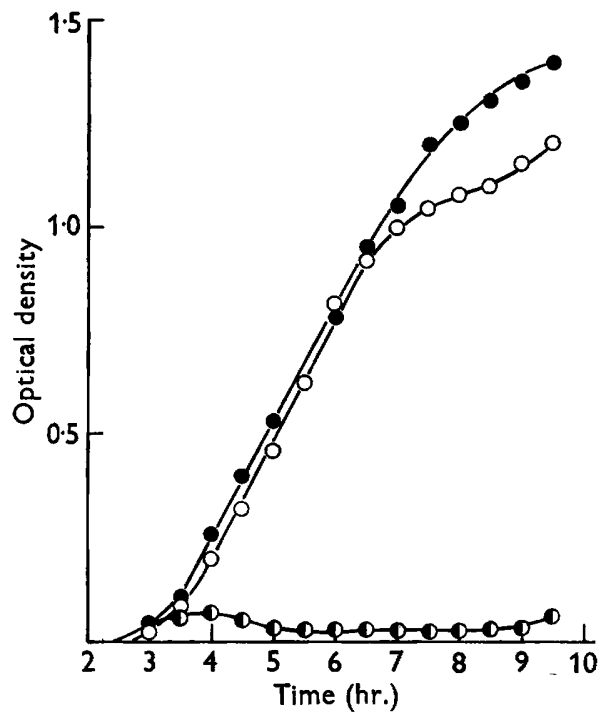

Fig. 1

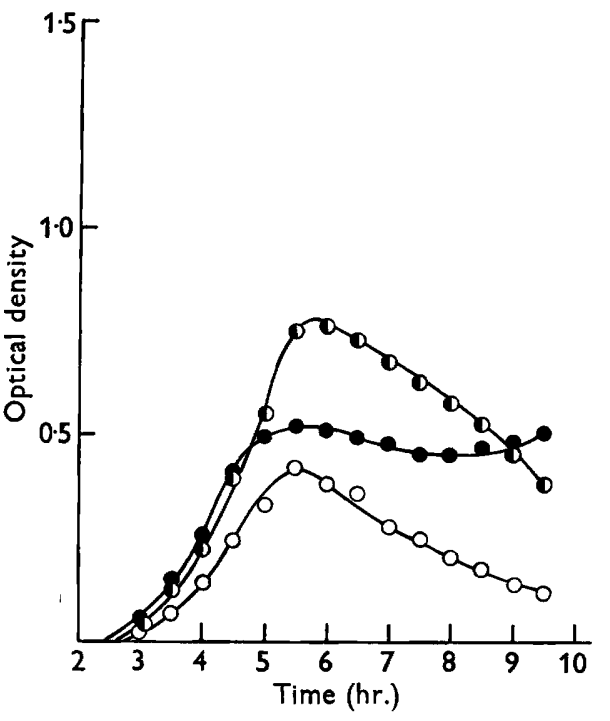

Fig. 2

Fig. 1. Growth of Bacillus megaterium strain 594 in various media. Media were inoculated from YDC agar. Size of inoculum : $3 \times 10^{6}$ colony-formers. Amount of medium: $10 \mathrm{ml}$. - meat extract; $\bigcirc-0$, YP; -0 , YDC.

Fig. 2. Growth of Bacillus megaterium strain 604 in various media. $\mathrm{O}-\mathrm{O}, \mathrm{YP} ; \mathbf{O}-\mathrm{D}, \mathrm{YDC}$.

Investigation of the aberrant strains for lysogeny. Although the indicator strain used in the screening test for iso-antagonistic effects was assumed to be entirely resistant to megaterium phages, we had also to consider whether the antagonistic effect and its enhancement by u.v. irradiation was due to a carried temperate phage. The difficulty in detecting small amounts of temperate phage in cultures of strains which may simultaneously elaborate megacin is that the latter kills the indicator strain when lysates or supernatant fluids of the cultures are assayed for infective centres. This was overcome by separating hypothetical phage from the culture by high-speed centrifugation of supernatant fluids, after sterilization by streptomycin, and subsequent assay for free phage by plating the deposit on a streptomycinresistant indicator strain.

Each of the 16 aberrant strains was examined. Cultures in YDC medium containing $2.5 \times 10^{8}-3.5 \times 10^{8}$ bacteria $/ \mathrm{ml}$. were first centrifuged at $3000 \mathrm{rev}$./ min. for $20 \mathrm{~min}$. to remove most of the bacteria. The supernatant fluids were 
collected and streptomycin (20 $\mu \mathrm{g}$. $/ \mathrm{ml}$. suspension) added to prevent the growth of residual bacteria. Six ml. of supernatant fluid were then centrifuged at $100,000 \mathrm{~g}$ for $1 \mathrm{hr}$., the supernatant fluid discarded and the pellet resuspended in YP medium. The suspension was again centrifuged at $100,000 \mathrm{~g}$ for $1 \mathrm{hr}$., and the pellet suspended in $6 \mathrm{ml}$. YP medium containing streptomycin. One ml. of each sample was then plated with a streptomycin-resistant phagesensitive indicator strain (Bacillus megaterium, strain $337 \mathrm{a}, 337 \mathrm{~b}$ or $\mathrm{KM}$ ) in an agar layer containing streptomycin. Fourteen of the 16 aberrant strains did not produce any plaques; that is, $c .10^{9}$ organisms of these strains did not yield even one infective phage particle. Two strains (508 and 597) proved to be lysogenic and gave small numbers of infective centres, the observed yield being 1 plaque from 50 bacteria of strain 508 or from 400 bacteria of strain 597. The efficiency of the above method was checked by treating a suspension of the megaterium phage $M_{1}\left(10^{10}\right.$ particles $/ \mathrm{ml}$. $)$ in the same way; $98 \%$ of the original phage was recovered. These results show clearly that most aberrant, that is megacinogenic, strains are non-lysogenic. Two strains yielded small numbers of plaque-forming particles, however, so that lysogeny and megacinogeny can occur simultaneously in some strains.

Table 2. The rate of growth and lysis expressed in optical density values of irradiated and control cultures of 17 strains of Bacillus megaterium in $Y D C$ medium

Young exponentially growing cultures at an optical density (OD) of 0.2 were u.v.irradiated. After re-incubation the extent of residual growth is expressed by the peak value $O D$, and the extent of lysis is expressed by the OD values measured at the end of experiment. The antibacterial titre is expressed by the reciprocal of highest effective dilution of supernatant fluid. Zero means no antibacterial effect undiluted material.

\begin{tabular}{|c|c|c|c|c|c|c|}
\hline \multirow{3}{*}{ Strain } & \multicolumn{3}{|c|}{ Irradiated cultures } & \multicolumn{3}{|c|}{ Non-irradiated culture } \\
\hline & $\begin{array}{l}\text { Peak } \\
\text { value }\end{array}$ & $\begin{array}{c}\text { At the } \\
\text { end of } \\
\text { experiment }\end{array}$ & $\begin{array}{l}\text { Anti- } \\
\text { bacterial } \\
\text { titre }\end{array}$ & $\begin{array}{l}\text { Peak } \\
\text { value }\end{array}$ & $\begin{array}{c}\text { At the } \\
\text { end of } \\
\text { experiment }\end{array}$ & $\begin{array}{c}\text { Anti- } \\
\text { bacterial } \\
\text { titre }\end{array}$ \\
\hline & \multicolumn{2}{|c|}{$\begin{array}{l}\text { Rel. optical density } \\
\text { values }\end{array}$} & & \multicolumn{2}{|c|}{$\begin{array}{l}\text { Rel. optical density } \\
\text { values }\end{array}$} & \\
\hline 216 & $0 \cdot 56$ & 0.04 & 40,000 & $1 \cdot 10$ & $1 \cdot 10$ & $\mathbf{0}$ \\
\hline 507 & 0.35 & 0.04 & 100 & $1 \cdot 40$ & $1 \cdot 40$ & $\mathbf{0}$ \\
\hline 508 & $0 \cdot 26$ & $0 \cdot 04$ & 10 & $1 \cdot 20$ & $1 \cdot 20$ & $\mathbf{0}$ \\
\hline 519 & $\mathbf{0 . 5 6}$ & $\mathbf{0 \cdot 1 3}$ & 100 & & & \\
\hline 527 & $0 \cdot 25$ & 0.04 & 1 & $1 \cdot 30$ & $1 \cdot 30$ & 0 \\
\hline 575 & $0 \cdot 50$ & $0 \cdot 20$ & 10 & $1 \cdot 30$ & $\mathbf{1} \cdot \mathbf{3 0}$ & $\mathbf{0}$ \\
\hline 594 & & & & $0 \cdot 20$ & 0.07 & 100 \\
\hline 597 & $0 \cdot 50$ & $0 \cdot 15$ & 10 & $1 \cdot 30$ & $\mathbf{1} \cdot \mathbf{3 0}$ & $\mathbf{0}$ \\
\hline 598 & 0.50 & $0 \cdot 11$ & 100 & $1 \cdot 30$ & $1 \cdot 30$ & $\mathbf{0}$ \\
\hline 604 & 0.27 & 0.09 & 10 & $0 \cdot 30$ & $0 \cdot 10$ & 1 \\
\hline 605 & $0 \cdot 28$ & $0 \cdot 03$ & 10 & $1 \cdot 40$ & $1 \cdot 40$ & $\mathbf{0}$ \\
\hline 637 & $\mathbf{0 \cdot 5 0}$ & $\mathbf{0} \cdot 13$ & 10 & $1 \cdot 35$ & $1 \cdot 35$ & $\mathbf{0}$ \\
\hline 638 & $0 \cdot 60$ & $0 \cdot 40$ & 1 & $1 \cdot 40$ & $1 \cdot 40$ & 0 \\
\hline 659 & 0.25 & 0.04 & 10 & $1 \cdot 05$ & $1 \cdot 05$ & 0 \\
\hline 669 & $0 \cdot 28$ & 0.04 & 10 & $1 \cdot 30$ & $1 \cdot 30$ & 0 \\
\hline 675 & 0.22 & 0.05 & 10 & $1 \cdot 20$ & $1 \cdot 20$ & 0 \\
\hline 681 & $\mathbf{0} \cdot 30$ & 0.08 & 10 & $1 \cdot 30$ & $1 \cdot 30$ & 0 \\
\hline 689 & 0.53 & $0 \cdot 17$ & 100 & $1 \cdot 40$ & $1 \cdot 40$ & 0 \\
\hline
\end{tabular}


Lysis induced by u.v. irradiation of cultures of Bacillus megaterium. Twenty ml. portions of YDC medium were inoculated from an overnight agar culture of the test strain and aerated by gentle shaking at $35^{\circ}$. When the viable count was $c .3 \times 10^{7} / \mathrm{ml}$., $10 \mathrm{ml}$. were placed in a Petri dish and irradiated with a highpressure lamp for $60 \mathrm{sec}$. The irradiated sample, and also a control un-irradiated culture, were then re-incubated. The megacin content of each culture was titrated at the end of each experiment. Table 2 shows that all the aberrant strains tested, lysed after irradiation and re-incubation. However, the amount

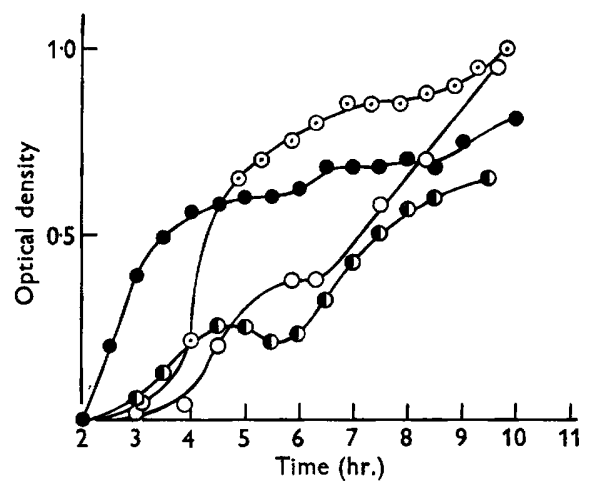

Fig. 8

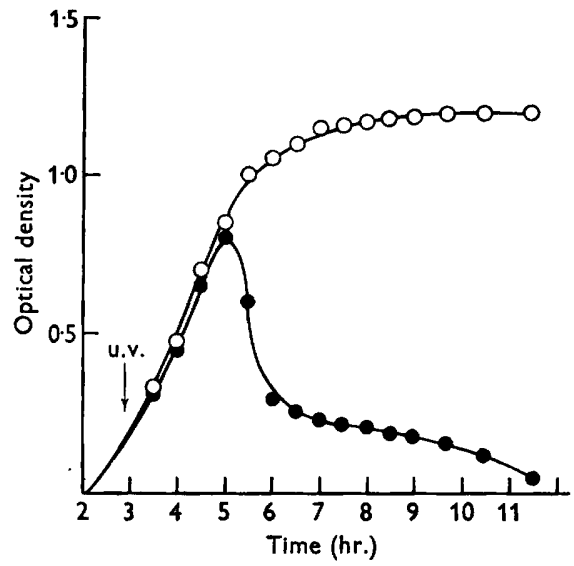

Fig. 4

Fig. 3. Growth of Bacillus megaterium strain 605 in horse meat extract broth in several experiments. Date of experiments: $\odot$, 30.v. 1956; $\odot$, 5. vi. 1956;, , 2. i. 1957; O, 16. v. 1957.

Fig. 4. Growth of Bacillus megaterium strain 216 and its 216 meg- mutant after u.v. irradiation. Megacin titres at the end of cultivation: 1/40,000 in lysate of 216. No antibacterial effect was shown by the supernatant fluid of strain $216 \mathrm{meg}$. $\mathrm{O}-\mathrm{O}$, strain 216; - , strain 216 meg-.

of residual growth, that is, the maximum optical density after irradiation, differed considerably with different strains. In some cases, the un-irradiated sample also showed early lysis (see strains 519 and 594 in Table 2). It must be stressed that early and apparently spontaneous lysis occurred more often when broth or YP medium was used instead of YDC medium. The megacin titres of cultures lysed by u.v. irradiation also differed enormously according to the strain. The titres appeared to be influenced also by the degree of lysis and by the maximum growth that was reached before lysis. When no detectable lysis occurred, as in the case of the un-irradiated of each culture, no megacin formation could be detected.

Several attempts were made to detect the presence of infective phage particles in lysates of irradiated cultures but only two of the strains mentioned above yielded phage, namely, 508 and 597, whose cultures contained respectively $2 \times 10^{6}$ and $5 \times 10^{8}$ plaque-forming particles $/ \mathrm{ml}$. A number of strains which did not show an iso-antagonistic effect in the screening test were also 
included in these experiments. None of them could be made to lyse by u.v. irradiation in various liquid media. Accordingly, no antibacterial effect was shown by supernatants of their cultures.

\section{Analysis of populations of megacinogenic strains}

General considerations. All the experiments described so far used inocula prepared from confluent growth on an agar slope and not from a single colony. The inocula were thus heterogeneous in origin. It should, however, be borne in mind that even when a suspension of vegetative Bacillus megaterium is used, colonies forming on solid media cannot be considered to be pure clones because of chain formation by this species. Homogeneous clones can only be obtained from a well-dispersed suspension of spores.

Spore suspensions were therefore used exclusively in the study of the genetic composition of the megacinogenic strains. Any vegetative organisms present $(c .5 \%)$ appeared to be degenerate, for their staining characteristics suggested advanced lysis. These remnants of non-sporing organisms have been disregarded below, whether or not they were capable of forming colonies. It would have been easy to get rid of these organisms by heat shock but this treatment was avoided because we feared that some spores of low heatresistance might be killed, so leading to a change in the composition of the population. Our aim has been to isolate non-megacinogenic mutants from strains which showed iso-antagonistic effects as well as aberrent growth when grown in liquid media. It was hoped that u.v. irradiation of stock spore suspensions would promote the appearance of such mutants, on the assumption that irradiation would lead to lysis of the megacinogenic members of the population (Ivánovics, Alföldi \& Lovas, 1957).

Any clone isolated from a megacinogenic strain which did not show an isoantagonistic effect when tested on indicator plates, even after u.v. irradiation, was designated ' $\mathrm{meg}^{-}$', provided this character remained stable on subculture. For example, $216 \mathrm{meg}^{-}$describes such a clone isolated from the megacinogenic strain 216. The identity of these clones was checked by their sensitivity to 'adapted' highly specific phages; or when these were not available, by the use of a set of megaterium phages differing in host range (Ivánovics, Alföldi \& Széll, 1957). Other markers used were the serological character of the cell wall (Ivánovics, 1955; Ivánovics, Alföldi \& Szell, 1957) and resistance to streptomycin. A non-megacinogenic mutant was accepted as a descendant of the test strain if it shared at least one marker character with the parent strain.

Experiments with strain 216. This strain is of particular interest because it is highly megacinogenic. Table 3 gives details of experiments carried out with 216, which was extremely sensitive to streptomycin, and with streptomycinresistant mutant of it, designated $216 \mathrm{~S}$, Both the mutant and parent stocks were attacked to the same extent by an 'adapted' phage. In 7 experiments, 8798 separate colonies were tested by replica-plating to indicator plates sown with a megacin-sensitive strain. Altogether, $\mathbf{5 2}$ colonies were found which did not produce an iso-antagonistic effect after small doses of u.v. radiation. These 


\section{Hereditary aberrancy in Bacillus megaterium}

strains were proved to be non-megacinogenic on further investigation. Thirtytwo non-megacinogenic colonies were isolated from strain $216 \mathrm{~S}^{\mathrm{r}}$ in the same way. All the non-megacinogenic strains possessed the same phage sensitivity, antigenic structure and resistance to streptomycin as the parent strain.

Table 3. Frequency of non-megacinogenic mutants in spore suspension of strains 216 and $216 \mathrm{Sr}$ of Bacillus megaterium

$\begin{array}{cllcc}\begin{array}{c}\text { No. of } \\ \text { experi- } \\ \text { ment }\end{array} & \text { Strain } & \text { Spore material } & \begin{array}{c}\text { No. of } \\ \text { colonies } \\ \text { examined }\end{array} & \begin{array}{c}\text { No. of } \\ \text { non-mega- } \\ \text { cinogenic } \\ \text { colonies }\end{array} \\ 1 & 216 & \text { Stock materìal } & 1167 & 2 \\ 2 & 216 & \text { 'u.v. spore' } 28 / 29 & 1016 & 0 \\ 3 & 216 & \text { 'u.v. spore' 55/61 } & 438 & 11 \\ 4 & 216 & \text { 'u.v. spore' } 69 & 985 & 7 \\ 5 & 216 S^{r} & \text { 'u.v. spore' 55/57 } & 994 & 2 \\ 6 & 2^{16 S} & \text { 'u.v. spore' 56/72 } & 1436 & 15 \\ 7 & 216 S^{r} & \text { 'u.v. spore' 56/74 } & 2762 & 15\end{array}$

The non-megacinogenic state has proved to be stable, for no back mutants have yet been detected during a large number of subcultures. In one experiment, one $216 \mathrm{meg}^{-}$isolate was kept in the logarithmic phase of growth by serial transfer to fresh medium. samples of the culture being tested at intervals by plating in an agar layer with megacin-sensitive cells; but no megacinogenic cells were isolated from the several hundred million cells cultivated.

None of the $\mathbf{5 2}$ non-megacinogenic isolates could be induced to lyse by exposure of cultures in liquid medium (YDC) to u.v. radiation. Nor did the supernatant fluids of irradiated cultures show any antibacterial effect when tested on indicator plates after re-incubation, thus proving a complete lack of megacin production by these cultures. Figure 4 illustrates the outcome of such an experiment. Another observation made during cultivation of the nonmegacinogenic mutants was that these invariably gave normal growth curves so that loss of megacinogeny appears to be paralleled by loss of an aberrant pattern of growth as well as by loss of inducibility by u.v. radiation.

Experiments with other megacinogenic strains. Experiments similar to those described above were carried out with 4 megacinogenic strains of different origin. Strain 597 , besides being megacinogenic, liberated a temperate phage during exponential growth, and lysis after u.v. irradiation was accompanied by mass liberation of phage particles. This strain, and also strain 575, were identified by their patterns of sensitivity to a set of Bacillus megaterium phages. In the case of strains 605 and 638 , highly specific 'adapted' phages were available for identification.

Table 4 shows that the proportion of non-megacinogenic isolates varied in different experiments. Two isolates were obtained from the lysogenic strain 597, one of which proved to have lost its lysogenicity, while the second carried the same phage as the parent strain. The lysogenic isolate was, however, no longer inducible by u.v. radiation.

A high proportion of non-megacinogenic mutants were isolated from strain 
605. No differences were found when either stock or 'u.v. spore' material was used. We consider that it is unjustifiable to conclude from the difference observed between the frequency of non-megacinogenic mutants obtained in these two experiments that u.v. irradiation promotes the isolation of these mutants.

Table 4. Frequency of non-megacinogenic mutants in spore suspension of individual megacinogenic strains of Bacillus megaterium

$\begin{array}{clcc}\text { Strain } & \text { Spore material } & \begin{array}{c}\text { No. of } \\ \text { colonies } \\ \text { examined }\end{array} & \begin{array}{c}\text { No. of } \\ \text { non-mega- } \\ \text { cinogenic } \\ \text { colonies }\end{array} \\ 575 & \text { 'u.v. spore' 56 } & 1746 & 5 \\ 597 & \text { 'u.v. spore' 54 } & 1065 & 0 \\ 597 & \text { 'u.v. spore' 56 } & 1033 & 2 \\ 605 & \text { Stock material } & 359 & 35 \\ 605 & \text { 'u.v. spore' 54 } & 714 & 63 \\ 638 & \text { Stock material } & 652 & 0 \\ 638 & \text { 'u.v. spore' } & 1086 & 22\end{array}$

Sensitivity of spores of the parent and the non-megacinogenic strains to u.v.irradiation. Although wild type organisms were lysed when their exponentially growing cultures were irradiated and re-incubated, lysis did not occur when spores of these strains were irradiated and plated on agar. Spore suspensions

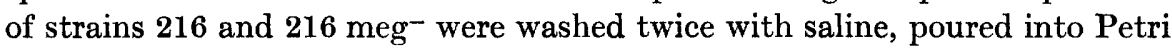
dishes to give a layer not more than $2 \mathrm{~mm}$. thick, and then irradiated with a low-pressure mercury lamp while the dish was gently swirled by hand. At intervals during irradiation, appropriate dilutions were plated on YP or on meat-extract agar and incubated. There was no significant difference between the survival curves of megacinogenic and of non-megacinogenic spores, both appearing to be equally sensitive to u.v. radiation. This implies that spores are not induced to lyse by u.v. radiation even when they are plated immediately after irradiation. The shapes of the curves suggest a multi-hit mode of killing. These results are in sharp contrast with our previous observations on the inducibility of exponentially-growing cultures (Ivánovics \& Alföldi, 1957).

\section{DISCUSSION}

Systematic investigations involving 200 strains of Bacillus megaterium recently isolated from soil provide further data concerning the occurrence of strains capable of producing an antibacterial principle identical or similar to that which has been described recently and named 'megacin' (Ivánovics \& Alföldi, 1954, 1955). Nearly half these strains elaborated megacin after exposure to small doses of u.v. radiation. The release of the antibacterial principle follows partial or complete lysis of the cultures and is enhanced or induced by the small doses of u.v. radiation acting on growing organisms. Strains which were highly inducible also displayed irregularities in their growth curves when cultivated in different complete media. It is supposed 


\section{Hereditary aberrancy in Bacillus megaterium}

that the aberrant growth pattern of these strains is elicited by some unknown agents which exist or appear during logarithmic growth in liquid media. Megacinogeny and aberrant growth pattern are therefore different manifestations of some factor occurring in a large proportion of Bacillus megaterium strains.

The behaviour of these aberrant strains is in many ways reminiscent of the behaviour of lysogenic bacteria. Nevertheless only 2 of 16 aberrant strains yield infective phage particles from their cultures; the remaining 14 strains did not yield even 1 infective particle $/ 10^{9}$ bacteria. Similarly, no infective particles could be demonstrated in cultures of megacinogenic strains after mass lysis had been induced by u.v. irradiation. Lysogeny and megacinogeny may coexist in the same bacterium as was shown in the case of 2 megacinogenic strains.

We think it can safely be said that megacinogeny is not necessarily associated with lysogeny, at least, not with a lysogenic relationship which results in the liberation of infective phage particles. However, the possibility of noninfective phage production is not excluded. A thorough study of strain 216, involving electron microscopy of lysing cells (Ivánovics, Alföldi \& Lovas, 1957), did not reveal any structures reminiscent of defective phage production. Such a study, however, only excludes incomplete phage production at a morphological level and does not disprove a possible synthesis of some nonorganized phage material governed by a highly degraded prophage.

As non-megacinogenic mutants were isolated from megacinogenic strains, it is safe to assume that there is a factor perpetuated in megacinogenic bacteria which governs the hereditary character of megacinogeny. This gene, whether located in the chromosomal or in the cytoplasmic substance of the organism, is responsible for phenomena such as inducibility to lysis, which in turn also shows itself by an aberrant pattern of growth in liquid media, as well as by megacin production. The genomes of Bacillus megaterium strains differ as to megacinogeny. Therefore, our observations may be of significance in the ecology of this species.

We wish to thank Dr Elinor W. Meynell for reading and correcting the manuscript.

\section{REFERENCES}

IvÁNovics, G. (1955). Dissociation of Bacillus megaterium associated with the change of the cell wall antigenic structure. Acta microbiol. Acad. Sci., Hung. 3, 135.

Ivánovics, G. \& AlföLdI, L. (1954). A new antibacterial principle: megacine. Nature, London, 174, 465.

IvÁNOvics, G. \& ALFöLDI, L. (1955). Observations on lysogenesis in Bacillus megaterium and on megacine, the antibacterial principle of this bacillus species. Acta microbiol. Acad. Sci., Hung. 2, 275.

Ivánovics, G. \& AlföldI, L. (1957). Bacteriocinogenesis in Bacillus megaterium. J. gen. Microbiol. 16, 522.

Ivánovics, G., Alfölddi, L. \& Lovas, B. (1957). Cultivation and electron microscopy of a bacteriocinogenic strain of Bacillus megaterium. Acta microbiol. Acad. Sci., Hung. 4, 295. 
Ivánovics, G., Alföldi, L. \& Széll, A. (1957). Serological types of Bacillus megaterium and their sensitivity to phages. Acta microbiol. Acad. Sci., Hung. 4, 333.

LeDerberg, J. \& Lederberg, E. M. (1952). Replica plating and indirect selection of bacterial mutants. J. Bact. 63, 399.

TARR, H. L. A. (1932). The relation of the composition of the culture medium to the formation of endospores by aerobic bacilli. J. Hyg., Camb. 32, 535.

(Received 22 April 1958) 